\title{
ACTUALIZACIÓN EN MATERIA DE CIRUGÍA ORTOPÉDICA Y TRAUMATOLO- GÍA DE LA REPERCUSIÓN CLÍNICA DE LA UTILIZACIÓN DE PRGF: PLASMA RICH IN GROWTH FACTORS
}

Salamanca Rodríguez D'., Corral Martinez C2.,Pastor Zaplana A²., Rico Ramirez V2., Escuder Capafons $M^{a} . C^{2}$

Objective: Update on plasma rich in growth factors (PRGF) in the field of orthopedic surgery and traumatology. Method: Review of 136 studies on PRGF relevant in the field of orthopedic surgery and traumatology published in the last 10 years. Results: The largest number of studies on PRGF has been performed in the field of osteoarthritis of the knee (25\%), followed closely by the comparative studies between PRGF and hyaluronic acid (19\%). The studies on ligamentous and tendinous pathology (14\%), the joint pathology different from the knee (10\%). The remaining studies addressed pseudoarthrosis, osteonecrosis and nerve regeneration among others. The most commonly used platelet products were Plasma Rich in Platelets (73\%), Plasma Rich in Growth Factors (15\%) and Plasma Rich in Fibrin (12\%). Conclusions: To document the preparation of platelet products, establish the precise concentration of growth factors and carry out long-term follow-up is essential for future studies.

Keywords: PRGF, PRP, Growth factors, Plasma rich in platelets, Plasma rich in growth factors, Plasma

${ }^{1}$ Corresponding Author: Daniel Salamanca Rodríguez danielsalamanca@live.com Cirugía Ortopédica y Traumatología Hospital de Manacor, C/Princep 26. CP:07500 Manacor, Espaก̃a

${ }^{2}$ Cirugía Ortopédica y Traumatología Hospital de Manacor, C/Princep 26. CP:07500 Manacor, España 


\section{INTRODUCCIÓN}

El proceso de regeneración tisular comprende un intrincado conjunto de acontecimientos biológicos equilibrados por la actuación sinérgica de varios factores de crecimiento. Tras la producción del daño tisular, se activan numerosas vías intracelulares e intercelulares con el propósito de restaurar la integridad del complejo tejido celular. Es bien conocido que los agentes implicados en la regeneración titular son: la matriz o "scaffold" que ofrece soporte al nuevo tejido en construcción, el componente celular y múltiples mediadores biológicos entre los que se incluye citoquinas y factores de crecimiento, necesarios para promover la angiogénesis que suministrará oxígeno y nutrientes al tejido dañado.

Las abreviaturas anglosajonas PRP (Plasma Rich in Platelets) y PRGF (Plasma Rich in Growth Factors) hacen referencia a todos aquellos procedimientos clínicos que mediante el centrifugado de la propia sangre del paciente logran aislar un concentrado de plaquetas o factores de crecimiento, el cual puede ser utilizado en ciertas intervenciones médico-quirúrgicas con el fin de acelerar los procesos de regeneración tisular.

Los factores de crecimiento que encontramos en este material biológico autólogo desempeñan una importante función en la comunicación intercelular, llevando a cabo un elevado número de funciones biológicas entre las que destaca la angiogénesis, proliferación celular, diferenciación, migración e incluso apoptosis.
El progreso en el estudio de las posibles aplicaciones terapéuticas de los factores de crecimiento ha permitido aumentar muy significativamente la versatilidad del uso clínico del Plasma Rico en Factores de Crecimiento.

\section{MATERIAL Y MÉTODOS}

En la presente revisión bibliográfica sobre la actualización en materia de plasma rico en factores de crecimiento, la búsqueda de la literatura fue realizada por un médico residente de cirugía ortopédica y traumatología. Los conflictos didácticos y técnicos se resolvieron mediante discusión y consulta a un segundo autor de la revisión. Se incluyeron todos los estudios publicados en materia de factores de crecimiento que evaluaron los diversos productos plaquetarios, independientemente del estado de la publicación o el idioma.

\section{Estrategia de búsqueda}

Se incluyeron aquellos artículos relevantes en el campo de la cirugía ortopédica y traumatología publicados en los últimos diez años. Se creó una base de datos con los artículos importados. Fueron consultadas las siguientes bases de datos y bibliotecas electrónicas sobre estudios potencialmente relevantes: PubMed, MEDLINE, Cochrane.

Las palabras clave utilizadas para la identificación de los estudios potencialmente relevantes fueron las siguientes: "PRGF", "PRP", "Factor de crecimiento", "Plasma rich in platelets", "Plasma rico en plaquetas", "Plasma rich in growth factors", "Plasma rico en factores de crecimiento", "Plasma". 
Se evaluó de forma independiente los títulos, objetivos, métodos, resultados y conclusiones de todos los artículos seleccionados, eliminando todos aquellos estudios duplicados. Se utilizaron los siguientes criterios de inclusión y exclusión previamente determinados:

\section{Criterios de inclusión}

- El estudio debe centrarse en la utilización clínica de cualquiera de estos productos plaquetarios: P-PRP, L-PRP, P-PRF, L-PRF y PRGF.

- El estudio debe ser realizado por investigadores en materia de cirugía ortopédica y traumatología.

- El estudio debe haber sido publicado en los últimos diez años.

- El estudio debe de presentar uno de los siguientes diseños de investigación: ensayos clínicos aleatorizados, ensayos clínicos no aleatorizados, informe de un caso, revisión sistemática, metaanálisis.

\section{Criterio de exclusión}

- Los sujetos de estudio presentaban trastornos plaquetarios.

- Los productos plaquetarios utilizados se administraron a través de la inyección endovenosa.

- El estudio ha sido realizado por investigadores en materia diferente de cirugía ortopédica y traumatología.

- El estudio ha sido publicado con anterioridad a los últimos diez años.

- Diseños de investigación diferentes a los previamente descritos.

\section{RESULTADOS}

Atendiendo a los criterios de inclusión de nuestro trabajo y tras realizar una búsqueda sistemática de los artículos de interés, se confeccionó una base de datos con un total de 136 estudios, de los cuales 91 procedían de Medline, 42 de Pubmed y 3 de Cochrane.

El mayor número de estudios sobre productos plaquetarios se ha realizado en el campo de la osteoartritis de rodiIla (25\%), seguido de cerca de los trabajos comparativos entre PRGF y ácido hialurónico $(\mathrm{AH})$ (19\%). Los trabajos que abordan la patología ligamentosa y tendinosa, así como la repercusión del tratamiento de las mismas con factores de crecimiento, representan el $14 \%$ de nuestra base de datos. La patología articular, deportiva o no, estudiada en una articulación diferente de la rodilla acumula el $10 \%$ de los trabajos. El 32\% de los estudios restante publicados bajo la especialidad médica de cirugía ortopédica y traumatología, se dividen en campos de acción tan dispares como la pseudoartrosis, osteonecrosis y regeneración nerviosa entre otros.

Los productos plaquetarios más utilizados en la bibliografía consultada fueron PRP (73\%), PRGF (15\%) y PRF (12\%).

\section{DISCUSIÓN}

El conocimiento del correcto uso de los factores de crecimiento es fundamental para avanzar en el manejo de la patología osteoarticular. Mediante la búsque- 
da y el posterior análisis de artículos relevantes en el campo de la cirugía ortopédica y traumatología publicados en los últimos diez años en materia de PRGF, se ha creado una base de datos para alcanzar información que nos permitiera conocer los recientes avances en relación a factores de crecimiento, implicados en la evolución clínica de la patología del aparato locomotor.

El equipo de trabajo del Dr. Yu Taniguchi [1] demostraron al igual que Anitua E. et al [2] una disminución de al menos el $50 \%$ del dolor según la Escala Analógica Visual (EVA) en el $80 \%$ de los pacientes a estudio. El Dr. Vaquerizo obtuvo una eficacia y seguridad significativamente mayor para el grupo tratado con PRGF, tras llevar a cabo un ensayo clínico aleatorizado de tres inyecciones de PRGF frente a una de AH [3]. El PRGF mostró resultados superiores a corto plazo cuando se comparó con $\mathrm{AH}$ en el alivio de los síntomas de osteoartritis de rodilla leve a moderada [4]. Las tasas de éxito observadas en la quinta semana de estudio para la subescala de dolor alcanzaron el 33,4\% para el grupo PRGF y el $10 \%$ para el grupo $\mathrm{AH}$ [5]. En el tratamiento de las lesiones osteocondrales del astrágalo, fue mayor en todos los casos la respuesta clínica al PRGF, aunque no se alcanzaron diferencias significativas [6].

El potencial terapéutico del PRGF como terapia biológica se basa en su capacidad de modular la expresión génica de los condrocitos [7], estimulando la mi- gración de los fibroblastos tendinosos y sinoviales, así como mejorando las propiedades biológicas del $\mathrm{AH}$ [8], influyendo de ese modo en el microambiente anabólico de la sinovial, manteniendo la homeostasis articular, mejorando el movimiento y disminuyendo el dolor. Estos hallazgos favorables apuntan a considerar el PRGF como una terapia biológica para la osteoartritis de rodilla [9].

Los atletas sufren frecuentes esguinces de tobillo, beneficiándose potencialmente de inyecciones de PRP guiadas por ultrasonidos, con un menor tiempo de retorno a la competición, debido a un menor dolor residual y una mayor estabilidad de la sindesmosis articular [10]. Las propiedades bioinductivas del PRGF en las lesiones del complejo ligamentoso del tobillo, permiten su utilización como alternativa terapéutica a la cirugía convencional o bien como terapia coadyuvante a la misma puesto que acelera la curación de las lesiones traumáticas y mejora las condiciones postoperatorias [11]. Galán M. et al administraron PRGF adyuvante a la tenorrafia quirúrgica de la rotura del tendón del cuádriceps bilateral, obteniendo una rehabilitación precoz del aparato extensor [12].

En ocasiones la mejoría clínica en el tratamiento de lesiones tendinosas con factores de crecimiento no se acompaña de hallazgos significativos en pruebas de imagen, como es el caso del equipo de Dr. Valentí Azcárate, que 
habiendo obtenido una disminución del edema en la reconstrucción con PRGF del ligamento cruzado anterior, la resonancia magnética no demostró diferencias en la intensidad ni en la uniformidad del injerto, y no hubo mejoría clínica o dolorosa en comparación con el grupo control [13]. Un ensayo clínico llevado a cabo en la reparación artroscópica con PRGF del manguito de los rotadores, no respalda su utilización en esta lesión, puesto que no se observaron diferencias significativas en la cicatrización del manguito o mejoría en las funciones evaluadas en el examen clínico y radiológico al año de la intervención quirúrgica [14]. En contraposición, Sánchez M. et al demostraron cambios histológicos temporales durante el periodo postoperatorio de 6 a 24 meses de la maduración del injerto tendinoso tratado con PRGF, que resultó en una mayor remodelación en comparación con los injertos no tratados con factores de crecimiento [15]. El conocimiento actual apunta a un futuro en el que las terapias con derivados plaquetarios continuarán mejorando los enfoques convencionales existentes para el tratamiento de la patología osteoarticular en general y deportiva en particular [16].

Existen controversia en la utilidad del PRGF como tratamiento de patología subyacente en la articulación temporomandibular (ATM), que a pesar de ser un campo de intervención maxilofacial se ha considerado en la presente revisión por su potencial terapéutico en el hueso. El grupo de trabajo del Dr. Fernández Sanromán concluyó que el
PRGF no agrega ninguna mejoría significativa a los resultados clínicos a los 2 años después de la cirugía en pacientes con trastorno interno avanzado de la ATM [17]. En esta línea de trabajo, la inyección de PRGF después de la artroscopia es más efectiva que la de $\mathrm{AH}$ con respecto a la valoración del dolor [18]. A su vez es necesario indicar que no existen datos científicos publicados que respalden suficientemente un protocolo de tratamiento específico con factores de crecimiento en la osteonecrosis del mandibular [19].

En traumatología, un importante uso de factores de crecimiento es el abordaje terapéutico de la pseudoartrosis. El grupo de trabajo del Dr. Sanchez M. demostró que el PRGF puede mejorar la curación de las pseudoatrosis no hipertróficas, al describir en su estudio un tiempo promedio de curación con esta técnica de 4,9 meses (2-8 meses), frente a los 21 meses (9-46 meses) necesarios para la curación de las hipertróficas [20]. A su vez, es de enorme utilidad en cirugía ortopédica la infiltración de nervios periféricos con PRGF, donde se ha comprobado que la infiltración de factores de crecimiento en la parálisis del nervio peroneo, mejora la clínica del paciente disminuyendo la caída del pie [21].

Es bien conocida la categorización en cuatro grupos diferentes de los derivados plaquetarios atendiendo al contenido de leucocitos y fibrinógeno; PRP puro (P-PRP), PRP y leucocitos (L-PRP), 
PRP y fibrina (P-PRF) y PRP más leucocitos y fibrina (L-PRF) [22-25]. Magalon et al. describieron la clasificación de DEPA, atendiendo a la dosis de "Plaquetas" inyectadas, "Eficacia" de producción, "Pureza" del PRP y "Activación" de PRP [26]. Estudios comparativos entre PRP, PRGF y PRF, determinan que la textura del PRF y sus características biológicas muestran claramente una mayor versatilidad quirúrgica que el resto de derivados plaquetarios [27]. Por otro lado, Filardo $\mathrm{G}$. Et al afirman que aunque las inyecciones de PRP produjeron más dolor e inflamación con respecto a las de PRGF, los resultados clínicos evaluados entre ambos compuestos fueron similares [28]. El análisis inmunocitoquímico tras la aplicación de estos hemoderivados, ha demostrado que los marcadores angiogénicos y precursores de las células CD34 (+) y CD133 (+) se mantienen en el cultivo durante un mayor periodo de tiempo [29], lo que pudiera proporcionar un avance en la aplicación clínica del autotrasplante de células madre.

Al tratarse de un material biológico autólogo, la administración de PRGF no genera reacción inmune o alergia. En consecuencia, los efectos secundarios graves son poco frecuentes en la literatura, siendo los principales efectos adversos el dolor e infección. Dado que el efecto de este tipo de tratamiento está ligado a la liberación de componentes bioactivos por parte de las plaquetas, puede que el uso de antiinflamatorios no esteroideos (AINE) modifique la funcionalidad plaquetaria y con ello limitar la eficacia del tratamiento. En este sentido se han realizado estudios con el fin de comparar la función plaquetaria en PRP de personas que están tomando simultáneamente un AINE, en relación al de personas que no lo han tomado en los 15 días anteriores, lo que ha llevado a considerar que el PRP obtenido tras la toma de AINE es de menor calidad y puede afectar negativamente al efecto de regeneración que se intenta conseguir con este tratamiento [30-32].

El estudio e identificación de los factores de crecimiento juega un papel trascendental en la reparación tisular. Múltiples trabajos refuerzan la importancia de los derivados plaquetarios en la patología osteoarticular, defendiendo la necesidad de protocolizar la preparación y aplicación de los mismos. Las terapias biológicas, han impulsado la investigación en cirugía ortopédica y traumatología hacia el descubrimiento de factores de crecimiento, y por qué no, nuevas estrategias de tratamiento. La optimización de la composición y el uso de PRGF es necesario para mejorar nuestro conocimiento en el potencial terapéutico de esta tecnología. Numerosos datos muestran que los efectos biológicos inducidos por los factores de crecimiento pueden depender de la concentración plaquetaria y de la fuente anatómica de las células [33]. El próximo paso científico en el saber de la regeneración tisular de la patología del aparato locomotor sería relacionar con notable evidencia científica dichos hallazgos clínicos con la aplicación local de PRGF. 


\section{CONCLUSIONES}

A pesar de que las investigaciones recientes abordan esta temática con especial interés científico, existe una carencia estadística respecto a la aplicación clínica de los factores de crecimiento. Progresivamente la calidad de la investigación está mejorando, y los campos de estudio sobre productos plaquetarios se están expandiendo, lo que podría sentar las bases de aplicaciones clínicas de estos derivados.

La necesidad de protocolizar la preparación de los productos plaquetarios, establecer la concentración precisa de plaquetas y factores de crecimiento así como el seguimiento a largo plazo de los sujetos del estudio son los factores más importantes para futuros estudios.

\section{REFERENCIAS BIBLIOGRÁFICAS}

[1] Intra-articular platelet-rich plasma (PRP) injections for treating knee pain associated with osteoarthritis of the knee in the Japanese population: a phase I and Ila clinical trial. Nagoya J Med Sci. 2018 Feb;

80(1): 39-51. Yu Taniguchi, Tomokazu Yoshioka, Akihiro Kanamori, Katsuya Aoto, et al.

[2] Efficacy and safety of plasma rich in growth factors intra-articular infiltrations in the treatment of knee osteoarthritis. Arthroscopy. 2014 Aug;30(8):1006-17. Anitua

E, Sánchez M, Aguirre JJ, Prado R, Padilla S, Orive G.

[3] Comparison of intra-articular injections of plasma rich in growth factors (PRGF-Endoret) versus Durolane hyaluronic acid in the treatment of patients with symptomatic osteoarthritis: a randomized controlled trial. Arthroscopy. 2013 Oct;29(10):1635-43. Vaquerizo V, Plasencia MÁ, Arribas I, Seijas R, Padilla S, Orive G, Anitua E. 
[4] A randomized clinical trial evaluat-

ing plasma rich in growth factors (PRGF-

Endoret) versus hyaluronic acid in the

short-term treatment of symptomatic knee

osteoarthritis.Arthroscopy. 2012

Aug;28(8):1070-8. Sánchez M, Fiz N, Azofra

J, Usabiaga J, Aduriz Recalde E, et al.

[5] Intra-articular injection of an autol-

ogous preparation rich in growth factors

for the treatment of knee OA: a retrospec-

tive cohort study. Clin Exp Rheumatol.

2008 Sep-Oct;26(5):910-3. Sánchez M, Ani-

tua E, Azofra J, Aguirre JJ, Andia I.

[6] Platelet-rich plasma or hyaluronate

in the management of osteochondral le-

sions of the talus. Am J Sports Med. 2012

Mar;40(3):534-41. Mei-Dan O, Carmont MR,

Laver L, Mann G, Maffulli N, Nyska M.

[7] A biological therapy to osteoarthri-

tis treatment using platelet-rich plasma.

Expert Opin Biol Ther. 2013

Aug;13(8):1161-72. Anitua E, Sánchez M,

Orive G, Padilla S.
[8] Plasma rich in growth factors

(PRGF-Endoret) stimulates tendon and

synovial fibroblasts migration and im-

proves the biological properties of hyalu-

ronic acid. Knee Surg Sports Traumatol

Arthrosc. 2012 Sep;20(9):1657-65. Anitua E,

Sanchez M, De la Fuente M, et al.

[9] Infiltration of plasma rich in growth factors for osteoarthritis of the knee shortterm effects on function and quality of life.

Arch Orthop Trauma Surg. 2011

Mar;131(3):311-7. Wang-Saegusa A1, Cu-

gat R, Ares O, Seijas R, et al.

[10] Plasma rich in growth factors

(PRGF) as a treatment for high ankle sprain in elite athletes: a randomized control trial. Knee Surg Sports Traumatol Arthrosc. 2015 Nov;23(11):3383-92. Laver L, Carmont MR, McConkey $\mathrm{MO}$, et al. 
[11] Conservative treatment using plasma rich in growth factors (PRGF) for injury to the ligamentous complex of the ankle. Acta Chir Orthop Traumatol Cech. 2008 Feb;75(1):28-33. Frei R, Biosca FE, Handl M, Trc T.

[12] Adjuvant administration of plasma rich in growth factors for bilateral quadriceps tendon tear. Acta Ortop Mex. 2014 Sep-Oct;28(5):310-4. Galán M, Seijas R, Ares O, Cuscó X, Rius M, Cugat R.

[13] Comparison between two different platelet-rich plasma preparations and control applied during anterior cruciate ligament reconstruction. Is there any evidence to support their use? Injury. 2014 Oct;45 Suppl 4:S36-41. Valentí Azcárate A, LamoEspinosa J, Aquerreta Beola JD, et al. [14] Plasma rich in growth factors in arthroscopic rotator cuff repair: a randomized, double-blind, controlled clinical trial. Arthroscopy. 2013 Jan;29(1):2-9. RuizMoneo P, Molano-Muñoz J, Prieto E, Algorta J.
[15] Ligamentization of tendon grafts treated with an endogenous preparation rich in growth factors: gross morphology and histology. Arthroscopy. 2010 Apr;26(4):470-80. Sánchez M, Anitua E, Azofra J, Prado R, Muruzabal F, Andia I. [16] Platelet-rich therapies in the treatment of orthopaedic sport injuries. Sports Med. 2009;39(5):345-54. Sánchez M, Anitua E, Orive G, Mujika I, Andia I.

[17] Does injection of plasma rich in growth factors after temporomandibular joint arthroscopy improve outcomes in patients with Wilkes stage IV internal derangement? A randomized prospective clinical study. Int J Oral Maxillofac Surg. 2016 Jul;45(7):828-35. Fernández Sanromán J, Fernández Ferro $M$, et al. 
[18] Comparison of intra-articular injec-

tion of plasma rich in growth factors versus hyaluronic acid following arthroscopy in the treatment of temporomandibular dysfunction: A randomised prospective study. J Craniomaxillofac Surg. 2017 Apr;45(4):449-454. Fernández-Ferro M, Fernández-Sanromán J, Blanco-Carrión A, Costas-López A, López-Betancourt A, et al. [19] Medication-related osteonecrosis of the jaw: Is autologous platelet concentrate application effective for prevention and treatment? A systematic review. J Craniomaxillofac Surg. 2016 Aug;44(8):106772. Lopez-Jornet P, Sanchez Perez A, Amaral Mendes R, Tobias A.

[20] Nonunions treated with autologous preparation rich in growth factors. J Orthop Trauma. 2009 Jan;23(1):52-9. Sanchez M, Anitua E, Cugat R, Azofra J, GuadiIla J, Seijas R, Andia I.

[21] Ultrasound-guided platelet-rich plasma injections for the treatment of common peroneal nerve palsy associated with multiple ligament injuries of the knee. Knee Surg Sports Traumatol Arthrosc. 2014 May;22(5):1084-9. Sánchez M, Yoshioka T, Ortega M, Delgado D, Anitua E. [22] Classification of platelet concentrates: from pure platelet-rich plasma ( $\mathrm{P}$ PRP) to leucocyte- and platelet-rich fibrin (L- PRF). Trends Biotechnol. 2009 Mar;27(3):158-67. Dohan Ehrenfest DM, Rasmusson L, Albrektsson T. [23] In search of a consensus terminology in the field of platelet concentrates for surgical use: platelet-rich plasma (PRP), platelet-rich fibrin (PRF), fibrin gel polymerization and leukocytes. Curr Pharm Biotechnol. 2012 Jun;13(7):1131-7. Dohan Ehrenfest DM, Bielecki T, Mishra A, Borzini $\mathrm{P}$, et al. 
[24] Morphogen and proinflammatory

cytokine release kinetics from PRGF-

Endoret fibrin scaffolds: evaluation of the

effect of leukocyte inclusion. J Biomed Ma-

ter Res A. 2015 Mar;103(3):1011-20. Anitua

E, Zalduendo MM, Prado R, Alkhraisat $\mathrm{MH}$,

Orive $\mathrm{G}$.

[25] Release kinetics of platelet-derived and plasma-derived growth factors from autologous plasma rich in growth factors. Ann Anat. 2013 Oct;195(5):461-6. Anitua E, Zalduendo MM, Alkhraisat MH, Orive G.

[26] DEPA classification: a proposal for standardising PRP use and a retrospective application of available devices. BMJ Open Sport Exerc Med. 2016 Feb 4;2(1):e000060. eCollection 2016. Magalon J, Chateau AL, Bertrand B, et al

[27] Comparison between PRP, PRGF and PRF: lights and shadows in three similar but different protocols. Eur Rev Med Pharmacol Sci. 2015;19(6):927-30. Giannini S, Cielo A, Bo- nanome L, Rastelli C, Derla C, Corpaci F, et al.

[28] Platelet-rich plasma intra-articular injections for cartilage degeneration and osteoarthritis: single- versus double- spinning approach. Knee Surg Sports Traumatol Arthrosc. 2012 Oct;20(10):2082-91. Filardo G, Kon E, Pereira Ruiz MT, Vaccaro F, et al.

[29] Platelet released growth factors boost expansion of bone marrow derived CD34(+) and CD133(+) endothelial progenitor cells for autologous grafting. Platelets. 2011;22(6):422-32. doi: 10.3109/09537104.2011.559559. Lippross S, Loibl M, Hoppe S, Meury T, et al.

[30] Autologous Platelet-Rich Plasma Preparations; Influence of Nonsteroidal Anti-inflammatory Drugs on Platelet Function. Journals SAGE, June 24, 2015. Gert Schippinger, Florian Prüller, Manuela Divjak, et al. 
[31] Effects of anti-aggregant, antiinflammatory and anti-coagulant drug consumption on the preparation and therapeutic potential of plasma rich in growth factors (PRGF). Growth Factors. 2015 Feb;33(1):57-64. Anitua E, Troya M, Zalduendo $\mathrm{M}$, Orive $\mathrm{G}$.

[32] The effect of different drugs on the preparation and biological outcomes of plasma rich in growth factors. Ann Anat. 2014 Dec;196(6):423-9. Anitua E, Troya M, Zalduendo MM, Orive G.

[33] Fibroblastic response to treatment with different preparations rich in growth factors. Cell Prolif. 2009 Apr;42(2):162-70. Anitua E, Sánchez M, Zalduendo MM, de la Fuente M, Prado R, Orive G, Andía I. 